\title{
GLOBAL EXISTENCE FOR THE MINIMAL SURFACE EQUATION ON $\mathbb{R}^{1,1}$
}

\author{
WILLIE WAI YEUNG WONG
}

(Communicated by Joachim Krieger)

\begin{abstract}
In a 2004 paper, Lindblad demonstrated that the minimal surface equation on $\mathbb{R}^{1,1}$ describing graphical timelike minimal surfaces embedded in $\mathbb{R}^{1,2}$ enjoy small data global existence for compactly supported initial data, using Christodoulou's conformal method. Here we give a different, geometric proof of the same fact, which exposes more clearly the inherent null structure of the equations, and which allows us to also close the argument using relatively few derivatives and mild decay assumptions at infinity.
\end{abstract}

\section{INTRODUCTION}

The equation describing graphical timelike minimal surfaces in $\mathbb{R}^{1, d+1}$ can be written as the quasilinear wave equation

$$
\left(\frac{m^{\mu \nu} \phi_{, \nu}}{\sqrt{1+m^{\sigma \tau} \phi_{, \sigma} \phi_{, \tau}}}\right)_{, \mu}=0
$$

for a real-valued function $\phi$, where $m^{\mu \nu}$ denotes the Minkowski metric on $\mathbb{R}^{1, d}$. In dimensions $d \geq 4$ the small data global existence follows largely from the linear decay of solutions to the wave equation which has the rate $\approx t^{-3 / 2}$, and is by now standard [Sog08]. In dimensions $d=2,3$, the linear decay of the wave equation has generic rates $\approx t^{-1 / 2}, t^{-1}$ respectively, which, not being integrable in time, can lead to finite-time singularity formation for even small data for generic quasilinear wave equations; see the survey article [HKSW16]. Equation (1) however exhibits the null condition, which is a structural condition on the nonlinearities identified first by Klainerman Kla86 and Christodoulou Chr86 in $d=3$ and later generalized by Alinhac in $d=2$ Ali01a, Ali01b. Using this fact Brendle Bre02 and Lindblad Lin04 established the small data global existence for (11) in dimensions $d=3$ and $d=2$ respectively. Brendle's proof followed the commuting vector field method of Klainerman Kla86. Lindblad, however, gave two different proofs in his paper using respectively the commuting vector field method as well as Christodoulou's compactification method.

In this paper we focus on the case $d=1$. The linear wave equation exhibits no decay in $d=1$, and hence the classical null condition cannot be used to assert that

Received by the editors January 6, 2016.

2010 Mathematics Subject Classification. Primary 35B35, 58J45.

The author thanks the Tsinghua Sanya International Mathematics Forum, Sanya, Hainan, People's Republic of China; as well as the National Center for Theoretical Sciences, Mathematics Division, National Taiwanese University, Taipei, Taiwan, for their hospitality during the period in which this research was performed. 
the perturbation is effectively short range, as in the case for $d \geq 2$. As a side effect this means that a direct proof of global existence for (11) in $d=1$ modeled after the commuting vector field method is not possible. A striking aspect of [Lin04] is that via the conformal compactification method, Lindblad was also able to prove the global existence of solutions to (11) in $d=1$ for small initial data. As Lindblad observed, the main trade-off is that for the conformal compactification method, the data must be of compact support, while in the vector field method the data is merely required to have "sufficiently fast" decay at infinity. The purpose of this paper is to produce an alternative proof of the $d=1$ case allowing initial data that is not necessarily compactly supported. In the course of the discussion we will also extract some more detailed geometric information concerning the solution when the data is of compact support.

\section{General geometric formulation}

The result of Brendle and Lindblad are based on comparing the timelike minimal surface to a flat hyperplane by normal projection, and the scalar function $\phi$ in (11) describes the deviation, or height, of the minimal surface as a graph over the hyperplane. The minimal surface equation can however be written intrinsically by way of the Gauss and Codazzi equations. To quickly recall: let $M \subseteq \mathbb{R}^{1, d+1}$ be a timelike hypersurface, then the Gauss equation requires that the Riemann curvature tensor 1 for the induced Lorentzian metric $g$ obey 2

(Gauss)

$$
\operatorname{Riem}_{a b c d}=k_{a c} k_{b d}-k_{a d} k_{b c}
$$

where $k$ is the second fundamental form of the embedding of $M$. The Codazzi equation on the other hand requires

$$
\text { (Codazzi) } \quad \nabla_{a} k_{b c}-\nabla_{b} k_{a c}=0
$$

where $\nabla$ is the Levi-Civita connection associated to $g$. It is well known that equations (Gauss and (Codazzil are the only obstructions to the existence of an isometric embedding. More precisely, we have the following theorem.

Theorem 1 (Fundamental theorem of submanifolds). Let $M$ be a simply-connected manifold, with a prescribed Lorentzian metric $g$ and a symmetric bilinear form $k$. Suppose that connection and curvature of $g$ satisfy (Gauss and (Codazzi). Then there exists an isometric immersion of $M$ into Minkowski space with one higher dimension, such that $k$ is the corresponding second fundamental form.

Remark 2. The Riemannian version of a more general theorem is well known. See for example Theorem 19 in Chapter 7 of [Spi79]. The Lorentzian version follows mutatis mutandis from the same proof. On the other hand, since we are considering the Cauchy problem, one can also simply "integrate" the second fundamental form in time once to obtain the normal vector field to $M$, and integrate it once more to get a parametrization of the hypersurface.

The upshot of this is that, the initial value problem for (1) can be reformulated as finding the metric $g$ and the second fundamental form $k$ from the initial data, requiring that they obey (Gauss) and (Codazzi).

\footnotetext{
${ }^{1}$ We use the convention Riem ${ }_{a b c}{ }^{d} X^{a} Y^{b} Z^{c}=\nabla_{[X, Y]} Z^{d}-\left[\nabla_{X}, \nabla_{Y}\right] Z^{d}$ which implies Ric $_{a c}=$ $\operatorname{Riem}_{a b c}{ }^{b}$.

${ }^{2}$ We will, throughout this paper, freely raise and lower indices using the induced metric.
} 
Equations (Gauss) and (Codazzi) do not yet describe an evolution: they are underdetermined. We get a well-posed initial value problem if we combine (Codazzi) with the minimal surface equation $\operatorname{tr}_{g} k=0$, which gives

(Minimality)

$$
\nabla^{a} k_{a b}=0 \text {. }
$$

Using that the Riemann curvature tensor is at the level of two derivatives of the metric, the initial data for the system (Gauss), Codazzil), and (Minimality consists of the metric $g$, the connection coefficients $\Gamma$, and the second fundamental form $k$ restricted to an initial slice. The initial value problem for (11) requires the data for $\phi$ and $\partial_{t} \phi$ at time $t=0$; these determine fully the first jet of $\phi$ restricted to the initial slice. As the equation (1) is second order hyperbolic, this formally provides us the $k$-jet of $\phi$ for any $k \in \mathbb{N}$ restricted to the initial slice, and a simple computation shows that the 2-jet of $\phi$ fully determines $g, \Gamma$, and $k$ on the initial slice. And thus we can indeed approach the vanishing-mean-curvature evolution through the initial value problem written in terms of the Gauss-Codazzi equations.

\section{Double null formulation when $d=1$}

For geometric partial differential equations, it is necessary to fix a gauge (i.e. make a coordinate choice). For hyperbolic equations it is convenient to use a double-null formulation. Since $M$ is a two-dimensional Lorentzian manifold, it is foliated by two transverse families of null curves. Equivalently, there exists two independent functions $u, v$ satisfying

$$
g(\nabla u, \nabla u)=g(\nabla v, \nabla v)=0
$$

and $g(\nabla u, \nabla v)>0$. Note that replacing $u$ by $f(u)$ for any $f: \mathbb{R} \rightarrow \mathbb{R}$ with $f^{\prime} \neq 0$ gives an equivalent reparametrization; we fix $u, v$ by prescribing their initial values on the initial slice, satisfying in particular that

$$
\text { Initial slice }=\{u-v=0\} .
$$

Since $u$ and $v$ are solutions to the eikonal equation (2), as is well known $L \stackrel{\text { def }}{=} \nabla u$ and $N \stackrel{\text { def }}{=} \nabla v$ are geodesic null vector fields, and at any point $\{L, N\}$ form a null frame of the tangent space $T_{p} M$.

The metric takes the form

$$
g=(\exp \psi)(\mathrm{d} u \otimes \mathrm{d} v+\mathrm{d} v \otimes \mathrm{d} u)
$$

where $\psi$ is some real-valued function. This implies that

$$
L(v)=g(\nabla u, \nabla v)=g(L, N)=N(u)=\exp (-\psi)
$$

so that the coordinate derivatives are

$$
\partial_{v}=(\exp \psi) L, \quad \partial_{u}=(\exp \psi) N
$$

and that the inverse metric is

$$
g^{-1}=\exp (-\psi)\left(\partial_{u} \otimes \partial_{v}+\partial_{v} \otimes \partial_{u}\right)=(\exp \psi)(L \otimes N+N \otimes L) .
$$

We can also compute

$$
\begin{aligned}
& \nabla_{L} N=(\exp \psi) g\left(L, \nabla_{L} N\right) N=-L(\psi) N \\
& \nabla_{N} L=(\exp \psi) g\left(N, \nabla_{N} L\right) L=-N(\psi) L .
\end{aligned}
$$


The assumption that $k$ is trace-free and symmetric requires that there exist scalar functions $\lambda, \nu$ such that

$$
k=\lambda L \otimes L+\nu N \otimes N .
$$

Then (Codazzi implies

$$
\left(L^{a} N^{b}-N^{a} L^{b}\right) \nabla_{a} k_{b c}=0
$$

and Minimality becomes

$$
\left(L^{a} N^{b}+N^{a} L^{b}\right) \nabla_{a} k_{b c}=0 .
$$

Taking linear combinations we get finally the null propagation equations for the second fundamental form

$(k-\mathrm{NP})$

$$
\begin{aligned}
& L(\lambda)=0, \\
& N(\nu)=0 .
\end{aligned}
$$

An immediate consequence of $(k-\mathrm{NP})$ is the following proposition.

Proposition 3. The scalar $\lambda$ is independent of $v$; the scalar $\nu$ is independent of $u$.

The evolution of the metric is now a scalar equation for the conformal factor $\psi$. The equation (Gauss implies

$$
\operatorname{Riem}_{a b c d} L^{a} N^{b} L^{c} N^{d}=\exp (-4 \psi) \lambda \nu .
$$

Combined with the definition that

$$
\operatorname{Riem}_{a b c d} L^{a} N^{b} L^{c} N^{d}=g\left(\nabla_{[L, N]} L-\left[\nabla_{L}, \nabla_{N}\right] L, N\right)
$$

we arrive at

$$
L(N(\psi))+L(\psi) N(\psi)=\exp (-3 \psi) \lambda \nu
$$

which we can rewrite as

$(\psi$-wave $)$

$$
\partial_{u v}^{2} \psi=\exp (-\psi) \lambda \nu .
$$

The equations $(k$-NP $)$ and $\psi$-wave can be regarded as evolution equations on a Minkowski space $\mathbb{R}^{1,1}$ (not the same one as the Minkowski space over which $\phi$ is a graph in the original setup; it is a manifold conformally equivalent to the graph itself with its induced Lorentzian metric) with $u, v$ the canonical null coordinates $r \pm t$, with initial data prescribed at $t=0$. In the remainder of this paper we discuss the easy consequences from the formulation above.

\section{Spatially COMPACT Initial DATA}

Suppose that the initial data for $\lambda, \nu$ is compactly supported, then for sufficiently large $u, v$ the functions $\lambda, \nu$ vanish respectively. In view of Proposition 3 and (9), we immediately see that

Theorem 4. For compact initial data, the manifold $M$ is intrinsically flat outside a compact domain.

Since $\psi$-wave is now a semilinear wave equation, with the nonlinearity only supported in a fixed space-time compact region, immediately by Cauchy stability we have

Theorem 5. For all sufficiently small compactly supported initial data, we have global existence for the initial value problem. 
Remark 6 . The compact support assumption is only necessary for $\lambda, \nu$, and not for $\psi$ !

Remark 7. One can easily check that the argument closes by standard energy arguments for initial data satisfying

- $\lambda, \nu \in L^{\infty}(\{t=0\})$ with compact support and sufficiently small norm;

- $\psi \in H^{1 / 2+\epsilon}(\{t=0\})$ and $\partial_{t} \psi \in L^{2}(\{t=0\})$ with sufficiently small norm.

The $H^{1 / 2+\epsilon}$ is for Sobolev embedding into $L^{\infty}$. The regularity outlined above is favorable compared to the classical local wellposedness level at $H^{5 / 2+\epsilon}$ for the quasilinear wave equation (1). Recalling that the second fundamental form is roughly two derivatives of the graphical function $\phi$ and the metric is at the level of $|\partial \phi|^{2}$, we see that the corresponding regularity for $\lambda, \nu \in H^{1 / 2+\epsilon}$ and $\psi \in H^{3 / 2+\epsilon}$ is higher compared to the result above.

\section{NONCOMPACT INITIAL DATA}

We can also handle the case where $\lambda, \nu$ are not compactly supported initially, but with sufficiently strong decay. This is the main theorem of this paper. Below we let $r=u+v$ be the coordinate function on the initial slice, and $t=u-v$ the time-function on $M ; \partial_{r}$ and $\partial_{t}$ refer to the coordinate derivatives in the $(r, t)$ coordinate system.

Theorem 8. Consider the initial value problem for the system $k$-NP and $\psi$-wave, with smooth initial data $\lambda_{0}, \nu_{0}, \psi_{0}=\left.\psi\right|_{\{t=0\}}$ and $\psi_{1}=\left.\partial_{t} \psi\right|_{\{t=0\}}$. Then there exists $\epsilon>0$ such that whenever the initial data satisfies

$$
\begin{gathered}
\left\|\lambda_{0}\right\|_{L^{1}}+\left\|\nu_{0}\right\|_{L^{1}} \leq \epsilon, \\
\left\|\psi_{0}\right\|_{L^{\infty}}+\left\|\partial_{r} \psi_{0}\right\|_{L^{1}}+\left\|\psi_{1}\right\|_{L^{1}} \leq \epsilon,
\end{gathered}
$$

we have a unique global solution.

Remark 9. As is well known, the regularity level $W^{1,1}$ is critical for wave equation in one spatial dimension.

Proof. Using ( $k$-NP and that $\lambda$ and $\nu$ propagate in different directions we immediately obtain that $\|\lambda \nu\|_{L_{t, r}^{1}} \approx\|\lambda \nu\|_{L_{u, v}^{1}} \lesssim \epsilon^{2}$. Using the fundamental solution for the wave equation in one dimension we get

$$
\|\psi(t, \cdot)\|_{L^{\infty}} \lesssim \epsilon+\|\lambda \nu \exp (-\psi)\|_{L^{1}\left([0, t]: L^{1}\right)} .
$$

This we bound by

$$
\epsilon+\exp \left(\|\psi\|_{L^{\infty}\left([0, t]: L^{\infty}\right)}\right) \cdot\|\lambda \nu\|_{L^{1}\left([0, t]: L^{1}\right)} \lesssim \epsilon+\epsilon^{2} \exp \left(\|\psi\|_{L^{\infty}\left([0, t]: L^{\infty}\right)}\right) .
$$

Then as long as $\epsilon$ is sufficiently small we have by bootstrapping that $\|\psi\|_{L_{t, r}^{\infty}}<3 \epsilon$. The theorem then follows from this a priori estimate and standard arguments.

\section{REFERENCES}

[Ali01a] S. Alinhac, The null condition for quasilinear wave equations in two space dimensions I, Invent. Math. 145 (2001), no. 3, 597-618, DOI 10.1007/s002220100165. MR.1856402

[Ali01b] S. Alinhac, The null condition for quasilinear wave equations in two space dimensions. II, Amer. J. Math. 123 (2001), no. 6, 1071-1101. MR.1867312

[Bre02] Simon Brendle, Hypersurfaces in Minkowski space with vanishing mean curvature, Comm. Pure Appl. Math. 55 (2002), no. 10, 1249-1279, DOI 10.1002/cpa.10044. MR 1912097 
[Chr86] Demetrios Christodoulou, Global solutions of nonlinear hyperbolic equations for small initial data, Comm. Pure Appl. Math. 39 (1986), no. 2, 267-282, DOI 10.1002/cpa.3160390205. MR820070

[HKSW16] Gustav Holzegel, Sergiu Klainerman, Jared Speck, and Willie Wai-Yeung Wong, Smalldata shock formation in solutions to $3 D$ quasilinear wave equations: an overview, J. Hyperbolic Differ. Equ. 13 (2016), no. 1, 1-105, DOI 10.1142/S0219891616500016. MR3474069

[Kla86] S. Klainerman, The null condition and global existence to nonlinear wave equations, Nonlinear systems of partial differential equations in applied mathematics, Part 1 (Santa Fe, N.M., 1984), Lectures in Appl. Math., vol. 23, Amer. Math. Soc., Providence, RI, 1986, pp. 293-326. MR837683

[Lin04] Hans Lindblad, A remark on global existence for small initial data of the minimal surface equation in Minkowskian space time, Proc. Amer. Math. Soc. 132 (2004), no. 4, 1095-1102 (electronic), DOI 10.1090/S0002-9939-03-07246-0. MR2045426

[Sog08] Christopher D. Sogge, Lectures on non-linear wave equations, 2nd ed., International Press, Boston, MA, 2008. MR2455195

[Spi79] Michael Spivak, A comprehensive introduction to differential geometry. Vol. IV, second ed., Publish or Perish, Inc., Wilmington, Del., 1979. MR.532833 (82g:53003d)

Department of Mathematics, Michigan State University, East Lansing, Michigan 48824

E-mail address: wongwwy@member.ams.org 